\title{
RhoA Regulates Dendrite Branching in Hippocampal Neurons by Decreasing Cypin Protein Levels
}

\author{
Hongxin Chen and Bonnie L. Firestein \\ Department of Cell Biology and Neuroscience, Rutgers University, Piscataway, New Jersey 08854-8082
}

\begin{abstract}
The way a dendrite is patterned determines how a neuron will receive information. The Rho GTPases have been reported to play increasingly well defined roles in determining dendritic branch and spine development and morphology. Much is known about how these small GTPases regulate the actin cytoskeleton; however, very little is known about how they alter the microtubule cytoskeleton. Our laboratory previously cloned and characterized cypin, a guanine deaminase that increases dendrite number by binding to tubulin heterodimers and promoting microtubule assembly. In the present study, we show that cypin and RhoA are part of a common pathway that regulates dendrite number. Inhibition of Rho kinase activity does not affect cypin-mediated dendrite branching. Furthermore, cypin does not affect the activity of RhoA, as measured by GTP binding to RhoA. In fact, activated RhoA acts to inhibit cypin protein expression and, by doing so, decreases dendrite number. In addition, this decrease in cypin protein occurs via a translation-dependent mechanism. Together, our data suggest that cypin acts downstream of the small GTPase RhoA to regulate dendrite branching in hippocampal neurons, providing a novel mechanism for RhoA action on microtubule dynamics.
\end{abstract}

Key words: RhoA; dendrite branching; hippocampal neurons; cypin; protein expression; signaling

\section{Introduction}

Neurons are highly polarized cells, most of which have a main axon that relays information to other neurons and dendrites that typically receive inputs from other neurons. The dendrites of neurons are often highly branched, and the extent of the arborization of the dendritic tree correlates with the number and distribution of inputs that the neuron can receive and process. A number of extracellular factors, such as neurotrophins (McAllister et al., 1995, 1997), netrins (Wadsworth, 2002), semaphorins (Dent et al., 2004), and Slits (Nguyen Ba-Charvet et al., 2001) have been reported to influence dendrite patterning. Similarly, there are recent reports of proteins, such as cypin (Akum et al., 2004), snapin (Chen et al., 2005), postsynaptic density-95 (PSD95) (Charych et al., 2006), $\beta$-catenin (Yu and Malenka, 2003), glutamate receptor-interacting protein 1 (Hoogenraad et al., 2005), and the phosphoinositide 3-kinase signaling pathway (Jaworski et al., 2005) that act as intracellular regulators of dendrite patterning. Neuronal activity also affects dendritic arbor development (Van Aelst and Cline, 2004).

One of the most studied intracellular regulators of dendrite arborization is the Rho family of small GTPases. This family includes Rho, Rac, and cell division cycle 42 (Cdc42), key regulatory molecules that link surface receptors to the organization of

Received Feb. 26, 2007; revised June 12, 2007; accepted June 25, 2007.

This work was supported in part by a Busch Biomedical grant, a New Jersey Governor's Council on Autism pilot grant, National Science Foundation Grants IBN-0234206 and IBN-0548543, March of Dimes Foundation Grant 1-FY04-107, and a grant-in-aid from the American Heart Association (B.L.F.). We thank Drs. Erik Charych and Yangzhou Du for comments on this manuscript.

Correspondence should be addressed to Dr. Bonnie L. Firestein, Department of Cell Biology and Neuroscience, Rutgers University, 604 Allison Road, Piscataway, NJ 08854-8082. E-mail: firestein@biology.rutgers.edu. DOI:10.1523/JNEUROSCI.0872-07.2007

Copyright $\odot 2007$ Society for Neuroscience $\quad$ 0270-6474/07/278378-09\$15.00/0 the actin cytoskeleton. These small GTPases have been shown to play increasingly important roles in determining dendritic branch and spine development and morphology (Arimura and Kaibuchi, 2005); however, there are conflicting reports on their action on dendrite outgrowth and branching (Redmond and Ghosh, 2001). In general, RhoA acts as a negative regulator of dendrite arbor growth. For example, overexpression of constitutively active mutants of RhoA (RhoCA) significantly reduces dendritic length in a number of organisms and cell types (Threadgill et al., 1997; Ruchhoeft et al., 1999; Lee et al., 2000; Li et al., 2000; Nakayama et al., 2000; Ahnert-Hilger et al., 2004; Pilpel and Segal, 2004). Conversely, expression of a dominantnegative mutant of RhoA (RhoDN) enhances dendritic development (Li et al., 2000; Wong et al., 2000; Ahnert-Hilger et al., 2004). However, there is a report that expression of RhoDN does not affect dendritic morphology in rat organotypic hippocampal slices (Nakayama et al., 2000). Thus, it still remains unclear how altering the activity levels of small GTPases, specifically RhoA, differentially affects dendrite branching in neurons.

We previously cloned and characterized cypin, a guanine deaminase that increases dendrite number by directly binding to tubulin heterodimers and promoting microtubule assembly (Firestein et al., 1999; Akum et al., 2004). RhoA activity regulates dendrite number and microtubule dynamics, two processes that are also regulated by cypin. Because the mechanism underlying RhoA action on the microtubule cytoskeleton in neurons is unknown, we examined whether RhoA and cypin act in the same pathway to regulate dendrite number.

In the present study, we show that cypin does not affect the activity of RhoA as measured by GTP binding to RhoA. In contrast, overexpression of a dominant-negative mutant of RhoA or inhibition of Rho with exoenzyme C3 transferase abolishes 
cypin-promoted increases in dendrite number in cultured hippocampal neurons. Inhibition of the RhoA effector Rho kinase (ROCK) has no effect on cypin-mediated dendrite branching. We also show that knocking down cypin protein expression with short hairpin RNA (shRNA) eliminates the effects of RhoA mutants on dendrite branching. Strikingly, overexpression of a constitutively active mutant of RhoA and activation of RhoA with lysophosphatidic acid (LPA) decrease cypin protein expression in hippocampal neurons. Interestingly, this regulation of cypin protein is not at the transcriptional level, but it is at the level of translation. Because cypin promotes microtubule assembly (Akum et al., 2004) and RhoA regulates cypin protein expression, we found a novel mechanism by which RhoA may act on the microtubule cytoskeleton and, hence, regulation of dendrite branching.

\section{Materials and Methods}

Antibodies and reagents. Rabbit anti-cypin (RTG-55) and preimmune serum were characterized previously (Akum et al., 2004). Rat anti-green fluorescent protein (GFP) was a kind gift from Dr. Shu-Chan Hsu of Rutgers University. Mouse anti-microtubule-associated protein 2 (MAP2) was purchased from BD PharMingen (San Diego, CA). Cyanine (Cy) 2-, Cy3- and Cy5-conjugated secondary antibodies were purchased from Jackson ImmunoResearch (West Grove, PA). Mouse anti-RhoA was purchased from Santa Cruz Biotechnology (Santa Cruz, CA). Exoenzyme C3 transferase was purchased from Cytoskeleton (Denver, CO). LPA, anisomycin, actinomycin D, MG132 [carbobenzoxy-L-leucyl-Lleucyl-L-leucinal], and monoclonal anti- $\beta$-actin antibody were purchased from Sigma (St. Louis, MO). Y27632 (4-[(1R)-1aminoethyl]- $N$-pyridin-4-yl-cyclohexane-1-carboxamide) was obtained from Calbiochem (San Diego, CA). Rho-binding domain (RBD)-glutathione $S$-transferase (GST) was a kind gift from Dr. Estela Jacinto (University of Medicine and Dentistry of New Jersey, Piscataway, NJ).

DNA constructs. Rho mutant constructs, T7-tagged V14RhoA (constitutively active) and N19RhoA (dominant negative), were subcloned into pEGFP-C1 and pDsRed-N1 vectors. Templates for these constructs for PCR were kind gifts from Dr. Anirvan Ghosh (University of California, San Diego, La Jolla, CA).

RhoA activity assay. The activity of RhoA was determined using the coprecipitation assays developed by Ren et al. (1999). Briefly, N2a cells were transfected with cDNA encoding GFP, GFP-cypin, or GFP-cypin mutants without guanine deaminase activity using Lipofectamine 2000 (Invitrogen, Carlsbad, CA) following the directions of the manufacturer. After $24 \mathrm{~h}, \mathrm{~N} 2 \mathrm{a}$ cells were washed with ice-cold PBS and lysed in lysis buffer $(50 \mathrm{~mm}$ Tris, $\mathrm{pH} 7.2,1 \%$ Triton X-100, 0.5\% sodium deoxycholate, $0.1 \%$ SDS, $500 \mathrm{~mm} \mathrm{NaCl}, 10 \mathrm{~mm} \mathrm{MgCl}, 10 \mathrm{mg} / \mathrm{ml}$ each of leupeptin and aprotinin, and $1 \mathrm{~mm}$ PMSF). Cells were further lysed by passing the extract through a 26 gauge needle 10 times. Cell lysates were clarified by centrifugation at $12,000 \times g$ at $4^{\circ} \mathrm{C}$ for $10 \mathrm{~min}$. Cell lysates were incubated with glutathione-Sepharose beads bound to $30 \mu \mathrm{g}$ of rhotekin RBD-GST fusion proteins for $1 \mathrm{~h}$ at $4^{\circ} \mathrm{C}$. Beads were washed three times with wash buffer ( $50 \mathrm{~mm}$ Tris, $\mathrm{pH} 7.2$, containing $1 \%$ Triton $\mathrm{X}-100,150 \mathrm{~mm} \mathrm{NaCl}, 10 \mathrm{~mm} \mathrm{MgCl}, 10 \mathrm{mg} / \mathrm{ml}$ each of leupeptin and aprotinin, and $0.1 \mathrm{~mm}$ PMSF). Bound RhoA proteins were resolved on a $15 \%$ SDS polyacrylamide gel and transferred to polyvinylidene difluoride (PVDF) membrane. Blots were probed with mouse anti-RhoA antibody. The total amount of RhoA in the cell lysate was used as a control for normalizing RhoA activity (level of GTP-bound RhoA) to total RhoA protein. Experiments were performed in triplicate.

Guanine deaminase activity assay. N2a cells were transfected with cDNA encoding GFP, GFP-cypin, or GFP-cypin mutants using Lipofectamine 2000 (Invitrogen) following the directions of the manufacturer. After $24 \mathrm{~h}, \mathrm{~N} 2 \mathrm{a}$ cells were washed with ice-cold PBS and scraped into $25 \mathrm{~mm}$ Tris- $\mathrm{HCl}, \mathrm{pH} 7.4$, and $150 \mathrm{~mm} \mathrm{NaCl}$. PMSF was added to a final concentration of $1 \mathrm{~mm}$. The cell suspension was homogenized using a Potter-Elvehjem tissue grinder (20 times). Lysates were then passed through a 25 gauge needle five times and centrifuged at $14,000 \mathrm{rpm}$ at $4^{\circ} \mathrm{C}$ for $15 \mathrm{~min}$. Samples (100 $\mu \mathrm{l}$ of lysate) were incubated with $900 \mu \mathrm{l}$ of either the negative control solution (1 $\mathrm{mm}$ 2,4,6-tribromo-3hyroxybenzoic acid, $0.1 \mathrm{~mm} 4$-amino-antipyrene, $0.025 \mathrm{U} / \mathrm{ml}$ xanthine oxidase, $0.00325 \mathrm{U} / \mathrm{ml}$ uricase, $0.002 \mathrm{U} / \mathrm{ml}$ peroxidase in $25 \mathrm{~mm}$ Tris$\mathrm{HCl}, \mathrm{pH} 7.4$, and $150 \mathrm{~mm} \mathrm{NaCl}$ ) or the assay solution (which contained all the aforementioned ingredients, including $0.5 \mathrm{~mm}$ guanine) at $37^{\circ} \mathrm{C}$ for $15 \mathrm{~min}$. Samples were centrifuged at $12,000 \times \mathrm{g}$ for $1 \mathrm{~min}$, and the optical density of each sample was measured at $512 \mathrm{~nm}$ after indicated time points.

Neuronal culture, immunocytochemistry, and transfection. Neuronal cultures were prepared from hippocampi of rat embryos at $18 \mathrm{~d}$ gestation. The hippocampi were dissociated by brief mechanical trituration. Cells were plated on poly-D-lysine-coated glass coverslips ( $12 \mathrm{~mm}$ diameter) at a density of $\sim 1800$ cells $/ \mathrm{mm}^{2}$. Cultures were plated and maintained in Neurobasal media supplemented with B27, penicillin, streptomycin, and L-glutamine. For immunocytochemistry, neurons were fixed in $4 \%$ paraformaldehyde in PBS for 15 min and labeled with the appropriate antibody. Labeled cells were visualized by immunofluorescence on an Olympus Optical (Tokyo, Japan) IX50 microscope with a Cooke Sensicam charge-coupled device cooled camera, fluorescence, imaging system, and Image Pro software (Media Cybernetics, Silver Spring, MD). For transfection, neurons were grown for $10 \mathrm{~d}$ in culture and transfected with the appropriate constructs using Effectene (Qiagen, Valencia, CA). Neurons were allowed to express the transfected protein for $48 \mathrm{~h}$ and then used for assay of dendrite number as we described previously (Akum et al., 2004; Chen et al., 2005). The experimenter was blinded to the condition when taking images and counting dendrite number. $n$ values include neurons from different coverslips and from two to three cultures derived from different embryos.

Sholl analysis was performed with a semiautomated program in $\mathrm{R}$, in which the soma boundary is approximated by an ellipsoid and dendrite intersections were assessed at radial distances from the soma (Charych et al., 2006). The dendritic tree was examined in $5 \mu \mathrm{m}$ increments. Statistical analysis was done with ANOVA followed by the appropriate post hoc test.

To test the effects of pharmacological inhibitors on dendrite number, neurons were transfected at $10 \mathrm{~d}$ in vitro (DIV 10) and treated at DIV 11 with either C3 transferase or Y27632 at the indicated concentrations for $24 \mathrm{~h}$. The experimenter was blinded to the condition when taking images and counting dendrite number.

For immunostaining, neurons were fixed in $4 \%$ paraformaldehyde in PBS for $15 \mathrm{~min}$. Cells were then incubated in blocking solution (PBS containing $0.1 \%$ Triton X-100, $2 \%$ normal goat serum, and $0.02 \%$ sodium azide) for $1 \mathrm{~h}$. All antibodies used were diluted in blocking solution. For GFP and MAP2 staining, dilutions of 1:500 for mouse anti-MAP2 and 1:1000 for rat anti-GFP were used. All incubations with primary antibodies were performed at room temperature on an orbital shaker for $2 \mathrm{~h}$. Coverslips containing the neurons were then washed with PBS three times. Secondary antibody consisted of a 1:250 dilution of Cy2conjugated donkey anti-rat $\operatorname{IgG}$ and Cy5-conjugated donkey antimouse IgG. All labeling with secondary antibodies was performed at room temperature on an orbital shaker for $1 \mathrm{~h}$. Washes were performed as described above. Coverslips were then mounted onto frosted glass microscope slides using Fluormount G (Southern Biotechnology, Birmingham, AL).

Quantification of fluorescence intensity. Hippocampal neurons were prepared, cultured, and transfected as above. The neurons were immunostained using our previously characterized cypin primary antibody (1:1000). Fluorescence intensities of cypin were measured using Image Pro software. Cell bodies for each neuron were traced, and intensities were measured as average pixel intensity within the selected region. Fluorescence was visualized using a $20 \times$ objective. To quantitate the fluorescence levels of tagged proteins, images of neurons were captured by CCD as above using a constant gain and exposure time for all samples. Images were corrected for coverslip fluorescence by subtracting a background image generated using an $11 \times 11$ erosion filter. The experimenter was blinded to the condition when taking images and assaying fluorescence intensities.

Western blotting. Hippocampal neurons were plated at $1 \times 10^{6}$ cells 
per $35 \mathrm{~mm}$ dish. At DIV 11, neurons were treated with either C3 transferase or Y27632 at the indicated concentrations for $24 \mathrm{~h}$. Neurons were washed with ice-cold PBS and lysed in lysis buffer (see above, RhoA activity assay). Cells were further lysed by passing the extract through a 26 gauge needle 10 times, and lysates were clarified by centrifugation at $12,000 \times g$ at $4^{\circ} \mathrm{C}$ for $10 \mathrm{~min}$. Proteins were resolved on a $15 \%$ SDS polyacrylamide gel and transferred to PVDF membrane. The blot was probed with the indicated antibodies. Experiments were repeated three or four times.

For quantitation of Western blotting, the blots were scanned and intensities of bands were quantitated using Adobe Photoshop software (Adobe Systems, San Jose, CA). An area close to the bands was used as a reference for background intensity. The difference between intensities of the background and the band is the absolute intensity of the band. The number of pixels for the bands was normalized to the intensity of the internal control ( $\beta$-actin) and then compared with that of the control condition. (i.e., no drug treatment or GFP expression) to yield normalized percentage intensity.

RNA interference. Small interference RNA targeting the cypin transcript (5'-CCCTCAAAGAAGTCTTCAG-3') and an unrelated sequence, as negative control ( $5^{\prime}$-GCTTGGTTACTCCTGGATT-3'), were converted to shRNA and subcloned into pSuper-GFP vector (Oligoengine, Seattle, WA) according to the instructions of the manufacturer. Oligonucleotides were synthesized and purchased from Bio-Synthesis (Lewisville, TX).

RNA interference rescue. A silent mutant of cypin (smCypin) was constructed using the QuikChange II Site-Directed Mutagenesis kit (Stratagene, La Jolla, CA). The cDNA encoding the silent mutant of cypin was cotransfected with cypin shRNA at DIV 10, and dendrite numbers were counted at DIV 12.

Real-time reverse transcription-PCR. Hippocampal neurons were treated with C3 transferase or LPA at DIV 10. Total RNA was isolated using Trizol reagent (Invitrogen). RNA was reverse transcribed with SuperScript II Reverse Transcriptase (Invitrogen). Relative quantitative real-time PCR was performed with Power SYBR Green PCR Master Mix (Applied Biosystems, Foster City, CA). The comparative cycle threshold Ct method ( $\Delta \Delta$ Ct method) was used for data analysis (Livak and Schmittgen, 2001).

\section{Results}

RhoA and cypin are part of a signaling pathway that regulates dendrite number

Both RhoA and cypin play important roles in dendrite branching. To further understand whether RhoA and cypin act as part of signaling pathway to regulate dendrite number, we overexpressed RhoCA and RhoDN with either GFP or GFP-cypin. Overexpression of RhoCA decreases primary and secondary dendrite number. When RhoCA and cypin were coexpressed, the numbers of primary and secondary dendrites were restored to basal levels (Fig. 1A,B). These data suggest that RhoA and cypin may act in an opposing manor to regulate dendrite number. Furthermore, overexpression of a dominant-negative mutant form of RhoA marginally increases primary dendrite number and significantly increases secondary dendrite number (Fig. $1 A, B$ ). Interestingly, coexpression of RhoDN with cypin results in an increase in secondary dendrite number similar to that of cypin alone. There is no additive effect on dendrite number, suggesting that cypin and RhoA are part of the same signaling pathway that regulates dendrite patterning (Fig. $1 A, B$ ).

We performed Sholl analysis by measuring the number of dendrites that cross ellipsoids at different radial distances from the cell body to analyze the roles of cypin and RhoA in higherorder dendritic branching (Fig. 1C). Sholl analysis shows similar results to proximal dendrite number as presented in Figure $1 B$. Overexpression of RhoCA decreases the number of intersections significantly, but coexpression with cypin blocks the effects of
A

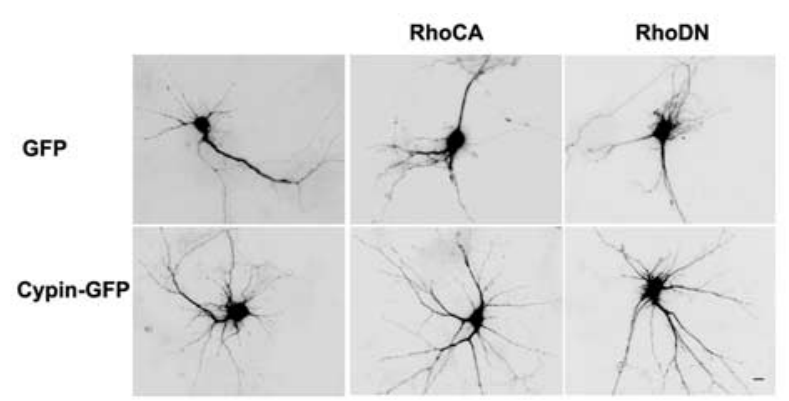

B
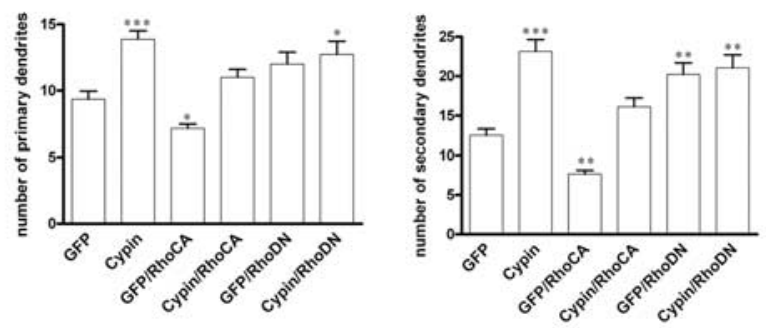

C
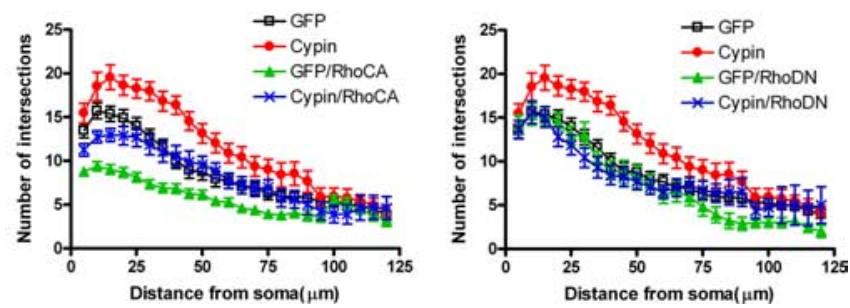

Figure 1. RhoA and cypin are part of a signaling pathway that regulates dendrite number. RhoCA and RhoDN were coexpressed with either GFP or GFP-cypin in hippocampal neurons from DIV 10-12. Neurons were fixed and immunostained for dendrite counting at DIV 12. $A$, GFP images of representative neurons. Scale bar, $10 \mu \mathrm{m}$. $\boldsymbol{B}$, Average number of primary and secondary dendrites in transfected neurons. $n$ values are as follows: GFP, $n=35$; cypin, $n=24$; GFP/RhoCA, $n=54$; cypin/RhoCA, $n=27$; GFP/RhoDN, $n=14$; cypin/RhoDN, $n=19$. * $p<$ $0.05,{ }^{* *} p<0.01,{ }^{* * *} p<0.001$ by Kruskal-Wallis test followed by Dunn's multiple comparisons test compared with GFP. C, Sholl analysis of transfected neurons. Overexpression of cypin (red circles) significantly increases number of intersections at 20-35 and $45 \mu \mathrm{m}$ from the soma compared with overexpression of GFP (black squares; $p<0.01$ at 25 and $30 \mu \mathrm{m}, p<0.05$ at other points). Left, 0verexpression of RhoCA (green triangles) dramatically decreases number of intersections at 5-40 $\mu \mathrm{m}$ from soma compared with overexpression of GFP (black squares; $p<0.05$ at $40 \mu \mathrm{m}, p<0.01$ at $20-30 \mu \mathrm{m}, p<0.001$ at other points). Expression of RhoCA blocks cypin-promoted increases in number of intersections, resulting in baseline (GFP) number of intersections. $p$ values were determined by Kruskal-Wallis test followed by Dunn's multiple comparisons test compared with GFP. Right, Coexpression RhoDN with GFP or cypin does not affect dendritic arbor complexity. Error bars indicate SEM.

RhoCA, resulting in no change from control (GFP). Although expression of RhoDN marginally increases primary dendrite number and significantly increases secondary dendrite number, Sholl analysis shows that expression of RhoDN alone or in combination with cypin does not change higher-order branching (Fig. 1C). Because Sholl analysis only counts dendrites that intersect concentric circles and does not differentiate between different orders of branching, this may be the reason why we see an increase in the number of proximal dendrites when RhoDN is expressed alone or in combination with cypin when analyzed by branch order but not by Sholl analysis (Fig. 1, $B$ vs $C$ ).

\section{Overexpression of cypin has no effect on RhoA activity in Neuro-2a cells}

Both cypin and RhoA activity can regulate dendrite branching and microtubule dynamics. In addition, cypin plays a role in 

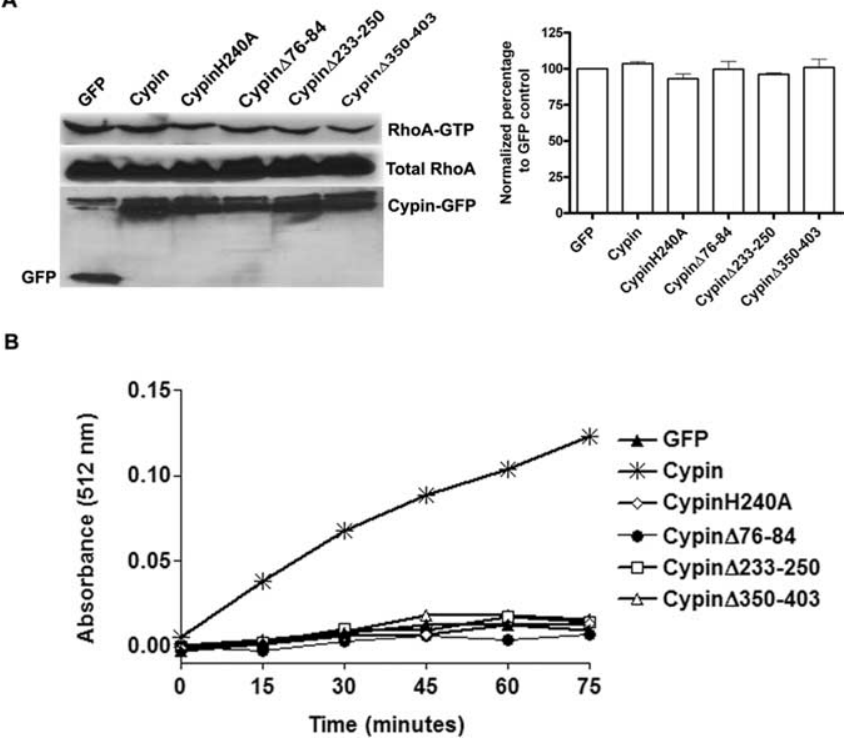

Figure 2. Overexpression of cypin does not affect RhoA activity in Neuro-2a cells. Neuro-2a cells were transfected with CDNA encoding GFP, cypin, or cypin mutants lacking guanine deaminase activity. A, Twenty-four hours after transfection, activated RhoA was isolated from cell lysates using rhotekin-GST coupled to glutathione-Sepharose beads. The eluates were subjected to Western blotting using an antibody to RhoA. Left, Representative blot is shown from three experiments. Right, Bands were quantitated as described in Materials and Methods. There is no significant change in the amount of RhoA activation when cypin or cypin mutants are expressed when compared with when GFP is expressed. $(n=3)$. $\boldsymbol{B}$, A colorimetric guanine deaminase activity assay was performed on Neuro-2a cells expressing GFP, cypin, or cypin mutants to confirm that the cypin mutants do not retain guanine deaminase activity $(n=3)$. Error bars indicate SEM.

guanine metabolism (Yuan et al., 1999; Akum et al., 2004), which may lead to alterations in GDP and GTP levels, thus affecting small GTPases, such as RhoA. To identify whether cypin increases dendrite branching by modulating RhoA activity, we transfected Neuro-2a cells, a mouse neuroblastoma cell line, with cDNA encoding cypin or cypin mutants and assayed RhoA activity. We found that overexpression of cypin does not change RhoA activity in these cells (Fig. 2A). Furthermore, mutants lacking guanine deaminase activity also do not affect RhoA activity (Fig. $2 A, B)$. Thus, our data suggest that cypin does not increase dendrite number by regulating RhoA activity.

Knockdown of cypin protein expression eliminates the effects of RhoA mutants on dendrite branching

Because cypin and RhoA appear to be part of a signaling pathway that regulates dendrite number, we asked whether cypin acts downstream of RhoA. We reasoned that if cypin is an effector of RhoA, knockdown of cypin protein would result in the loss of RhoDN-mediated increases in dendrite branching. Thus, we constructed cypin shRNA and an unrelated shRNA (control) using the pSUPER RNAi vector system. We transfected cultured hippocampal neurons on DIV 10 with pSUPER vector, control shRNA, or cypin shRNA. After $48 \mathrm{~h}$, the neurons were fixed and immunostained for cypin. We found that cypin shRNA attenuates cypin protein expression, whereas vector and control shRNA have no effect on cypin protein expression (Fig. $3 A$ ). We then quantitated the fluorescence intensity of cypin immunostaining of the transfected neurons. As shown in Figure 3B, cypin shRNA knocks down cypin levels significantly, whereas the control shRNA has no effect.

To demonstrate the specificity of our cypin shRNA construct,
A

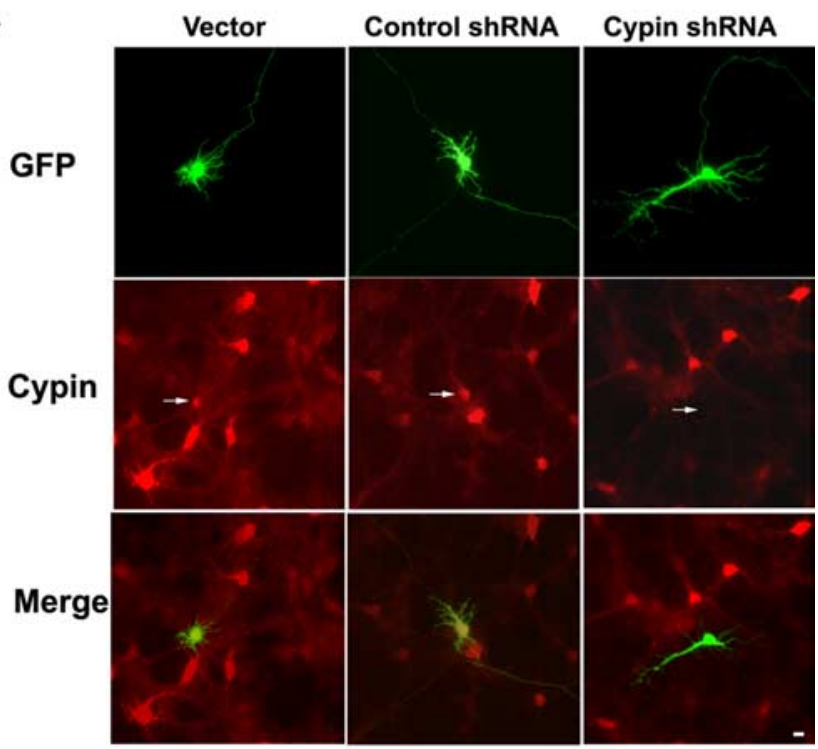

B

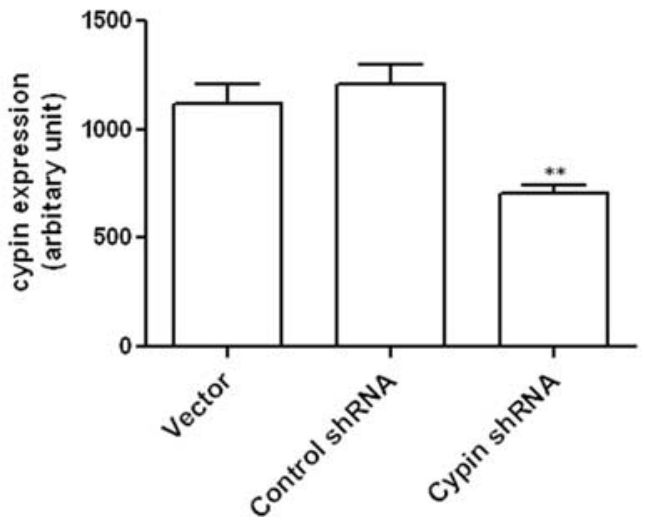

Figure 3. shRNA knocks down cypin protein expression. Hippocampal neurons were transfected with vector, control shRNA, or cypin shRNA at DIV 10. Neurons were fixed and immunostained for GFP and cypin at DIV 12. A, Representative GFP fluorescence images of neurons transfected with cDNAs encoding the stated constructs. Arrows in cypin panels (middle row) indicate neurons transfected with the stated cDNAs. Scale bar, $10 \mu \mathrm{m}$. B, After the cells were fixed and immunostained for cypin at DIV 12 , cypin immunoreactivity was measured using the ImagePro analysis program. $n$ values are as follows: vector, $n=45$; control shRNA, $n=34$; cypin shRNA, $n=49 .{ }^{* *} p<0.01$ by Kruskal-Wallis test followed by Dunn's multiple comparisons test compared with vector. Error bars indicate SEM.

we constructed an smCypin that cannot be targeted by our shRNA. When overexpressed in the presence of the cypin shRNA construct, this silent mutant should rescue cypin protein expression and function (Fig. 4A). As expected, dendrite number returned to basal levels when we coexpressed cypin shRNA with the cypin silent mutant (Fig. $4 B, C$ ).

To assess whether knocking down cypin protein expression affects RhoA regulation of dendrite branching, we expressed RhoCA or RhoDN in cells with cypin protein knocked down and counted dendrite numbers. Expression of cypin shRNA decreases dendrite number significantly (Fig. 5A,B). Expression of cypin shRNA concurrently with expression of RhoCA had no effect on RhoCA-promoted decreases in dendrite number (Fig. 5A,B). Transfection of the RhoDN construct alone increases dendrite number in neurons, but this increase in dendrite number was blocked when cypin shRNA but not control shRNA was coexpressed (Fig. $5 A, B$ ). Thus, knockdown of cypin protein expres- 
sion decreases dendrite number regardless of whether RhoCA or RhoDN is expressed (Fig. 5A,B).

Together, these data suggest that cypin acts downstream of RhoA in the signaling pathway regulating dendrite number in hippocampal neurons and that cypin may be an effector of RhoA activity.

\section{Cypin regulates dendrite branching in a ROCK-independent manner}

To determine how RhoA activity may regulate dendrite branching through cypin, we treated cultured hippocampal neurons with C3 transferase, a specific inhibitor of Rho GTPases. As seen in Figure 6, $A$ and $B$, treatment with $\mathrm{C} 3$ transferase had no effect on primary dendrite number; however, it significantly increased secondary dendrite number. Interestingly, this result differs slightly from what is seen with overexpression of RhoDN, in which both primary and secondary dendrite number increased (Fig. 1A,B). This could be attributable to two reasons. (1) RhoDN and $\mathrm{C} 3$ transferase act via different mechanisms to inactivate RhoA. RhoDN is RhoN19, which has higher affinity for GDP than GTP, and inhibits the action of guanine nucleotide exchange factors. C3 transferase causes ADP-ribosylation of RhoA, which renders RhoA inactive. (2) Because the transfection efficiency of neurons is low, RhoDN expression reflects a cell-autonomous effect, whereas C3 transferase treatment affects all cells in the dish, resulting in intrinsic and extrinsic effects. Furthermore, the increase in secondary dendrite number promoted by treatment with $\mathrm{C} 3$ transferase was not additive with the increase promoted by cypin overexpression (Fig. $6 A, B$ ), suggesting that inactive Rho and cypin are part of a shared pathway that regulates dendrite number. In contrast, treatment with Y27632, an inhibitor of the Rho effector ROCK, had no effect on cypin-promoted increases in dendrite number. Treatment with both inhibitors also suppresses the increase caused by cypin overexpression (Fig. 6A-C). In addition, Sholl analysis indicates that there is no increase in dendritic arbor complexity after treatment with C3 transferase, and Y27632 treatment has no effect on increases in dendrite number mediated by cypin (Fig. 6C). Treatment with Y27632 alone also increases arbor complexity distal to cell body, indicating that ROCK may affect higher-order dendrite branching (Fig. $6 C)$. Inhibition of both RhoA and ROCK drastically suppresses the effect of cypin (Fig. 6A-C). Again, Sholl analysis does not distinguish the branch order of dendrites and thus may obscure effects seen at individual branch orders (Fig. 6, $B$ vs $C$ ).

Western blotting of lysates from neuronal cultures treated with either C3 transferase or Y27632 showed that cypin protein expression is not significantly changed by these treatments (Fig. $6 D, E)$. Because our data suggest that RhoDN and $\mathrm{C} 3$ transferase increase dendrite number via a cypin-dependent mechanism, this may be achieved by posttranslational modification of cypin, leading to increased cypin activity. Together, these data suggest that cypin acts downstream of Rho in a ROCK-independent manner to regulate dendrite number.

\section{Activation of RhoA decreases endogenous cypin protein expression via a translation-dependent mechanism}

Because RhoA and cypin both regulate dendrite number, it is possible that RhoA activity may affect cypin protein expression, i.e., cypin may act downstream of RhoA. To test this hypothesis, we transfected hippocampal neurons at DIV 10 with cDNAs encoding constitutively active and dominant-negative forms of RhoA and measured cypin protein expression in individual neurons by immunostaining $2 \mathrm{~d}$ later (DIV 12). Overexpression of RhoCA in hippocampal neurons results in decreased endogenous cypin protein levels (Fig. $7 A$ ). In contrast, overexpression of RhoDN has no effect on cypin protein expression (Fig. 7A). Similarly, activation of RhoA with LPA significantly decreases cypin protein expression levels (Fig. 7B). LPA activates RhoA very quickly (Ren et al., 1999) and has been shown to specifically activate RhoA in neuronal cell lines and neurons (Gebbink et al., 1997; Li et al., 2000). It has also been reported that RhoA stimulates promoter activity in smooth muscle cells (Cui et al., 2006; Larsson et al., 2007). We asked whether activation of RhoA decreases cypin protein levels by altering transcription of cypin mRNA. To our surprise, LPA activation of RhoA does not affect cypin mRNA expression levels (Fig. 7C). Our data suggest that activation of RhoA decreases cypin protein through a transcription-independent mechanism. In fact, actinomycin D, 
A

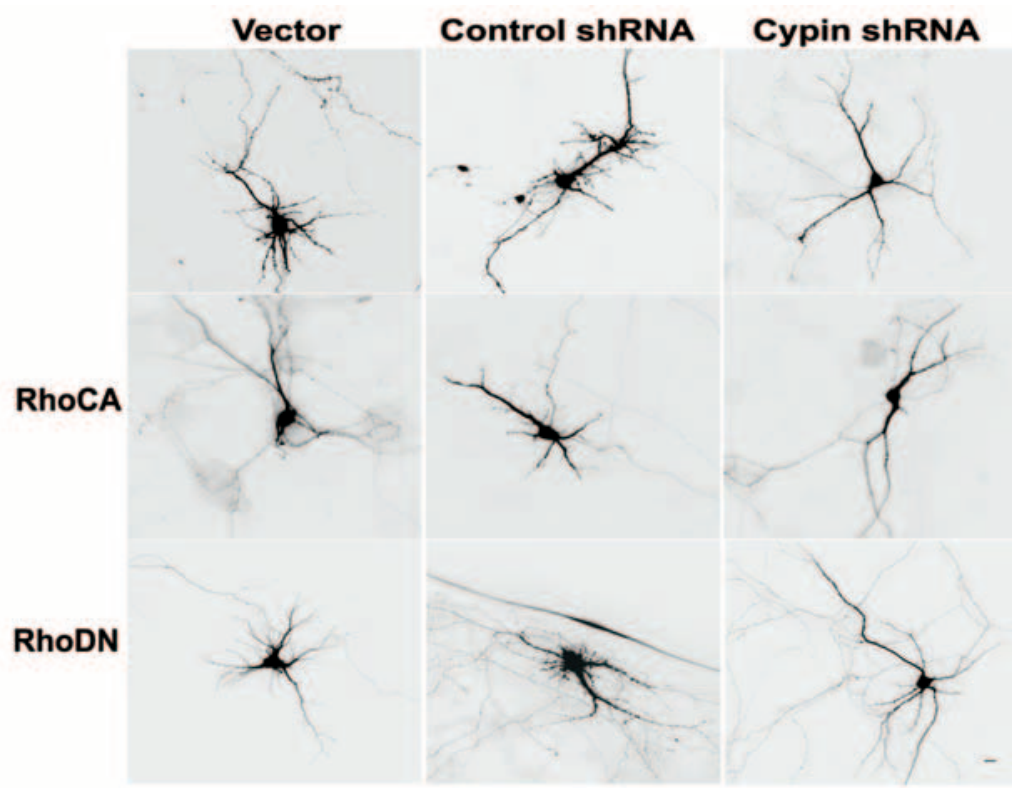

B
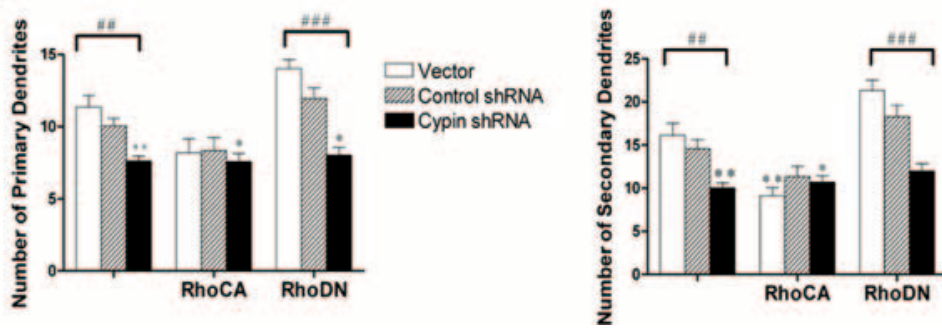

Figure 5. Knockdown of cypin protein expression eliminates the effects of RhoDN on dendrite branching. Hippocampal neurons were transfected with cDNAs encoding the stated constructs at DIV 10. Neurons were fixed and immunostained for dendrite counting at DIV 12. A, GFP images of representative neurons. Scale bar, $10 \mu \mathrm{m}$. $\boldsymbol{B}$, Average number of primary and secondary dendrites in transfected neurons. $n$ values are as follows: vector, $n=17$; control shRNA, $n=21$; cypin shRNA, $n=33$; RhoCA plus vector, $n=20$; RhoCA plus control shRNA, $n=18$; RhoCA plus cypin shRNA, $n=25$; RhoDN plus vector, $n=28$; RhoDN plus control shRNA, $n=32$; RhoDN plus cypin shRNA, $n=31 .{ }^{*} p<0.05,{ }^{* *} p<0.01$ and ${ }^{\# \#} p<0.01$, ${ }^{\# \# \#} p<0.001$ by KruskalWallis test followed by Dunn's multiple comparisons test compared with untreated vector unless otherwise indicated. Error bars indicate SEM.

an RNA synthesis inhibitor, does not block the RhoA-mediated decrease in cypin protein expression (Fig. $7 D, E$ ). To further elucidate the mechanism by which RhoA decreases cypin protein expression, we used a protein synthesis inhibitor, anisomycin, and a general proteasome inhibitor, MG132, to block translation and ubiquitin-dependent degradation, respectively. As seen in Figure 7, $D$ and $E$, anisomycin, but not MG132, blocked the LPAmediated decrease in cypin protein expression. This indicates that RhoA activity decreases cypin protein in a translationdependent manner, resulting in decreased dendrite number.

\section{Discussion}

Although many groups have reported that RhoA activation negatively regulates dendritic arbor growth (Ruchhoeft et al., 1999; Lee et al., 2000; Li et al., 2000; Nakayama et al., 2000; Wong et al., 2000; Ahnert-Hilger et al., 2004; Pilpel and Segal, 2004), little is known about the molecular mechanism underlying the influence of RhoA on dendrite branching. The main finding of this study is that RhoA acts to regulate dendrite number through a cypindependent pathway. We show that cypin does not affect the activity of RhoA. Furthermore, coexpression of a dominantnegative mutant of RhoA with cypin results in an increase in secondary dendrite number similar to that of cypin alone. Similarly, inhibition of Rho with exoenzyme C3 transferase results in increased secondary dendrite number that is not additive with increases mediated by cypin, suggesting that RhoA and cypin are part of a common signaling pathway. Knocking down cypin protein expression with shRNA eliminates the increases in dendrite number by inactive RhoA. Most excitingly, active RhoA acts to regulate dendrite number by decreasing cypin protein expression via a translationdependent mechanism.

We found that cypin acts downstream of RhoA in a ROCK-independent manner. The effects of RhoA on dendrite branching have been at least in part attributed to ROCK (Redmond and Ghosh, 2001; Govek et al., 2005; Newey et al., 2005), which plays an important role in actin cytoskeleton assembly and cell contractility (Riento and Ridley, 2003). Although treatment of rat hippocampal slices with the ROCK-specific inhibitor Y27632 effectively blocks the dendritic branch reduction induced by the constitutively active mutant of RhoA, neither overexpression of a dominant-negative mutant of RhoA nor treatment with Y27632 alone induces any detectable phenotype change (Nakayama et al., 2000). Exposure of Xenopus tadpoles to Y27632 increases the growth rate of tectal cell dendritic arbors similar to that of dominant-negative mutant of RhoA (Li et al., 2002; Sin et al., 2002). Our results suggest that RhoA activation mediates dendrite arbor simplification through a pathway that involves cypin.

Because cypin is a guanine deaminase and could ultimately alter intracellular concentrations of GTP, we hypothesized that cypin could alter RhoA activity to regulate dendrite branching in the neuron. However, we found this hypothesis to be incorrect because neither cypin nor cypin mutants lacking guanine activity change RhoA activity (Fig. 2). In fact, our data strongly suggest that cypin acts downstream of RhoA. What is the significance of this signaling pathway? Recently, it has been shown that Rho GTPases regulate microtubule dynamics (Zheng, 2004; Jaffe and Hall, 2005; Watanabe et al., 2005; Charest and Firtel, 2007). One of the few RhoA effectors reported to mediate the influence of RhoA on the microtubule cytoskeleton is the family of diaphanous-related formins ( $\mathrm{mDia}$ ). $\mathrm{mDia}$ acts to regulate microtubule alignment (Ishizaki et al., 2001) and the formation and orientation of stable microtubules (Ishizaki et al., 2001; Palazzo et al., 2001). However, the action of RhoA through mDia appears to be ROCK independent (Maekawa et al., 1999). In addition, the ROCK substrate collapsin response mediator protein-2 (CRMP-2) binds tubulin heterodimers and promotes microtubule assembly (Fukata et al., 2002). Remarkably, cypin contains a region of homology to CRMP that binds directly to tubulin heterodimers and promotes microtubule assembly in hippocampal neurons (Akum et al., 2004).

RhoA, Rac1, and Cdc42 are well characterized for their effects on the actin cytoskeleton. Activation of these GTPases results in 
the assembly of contractile actin:myosin filaments, protrusive lamellipodia, and protrusive filopodia, respectively, followed by actin polymerization and filament bundling (Hall, 1998; EtienneManneville and Hall, 2002; Luo, 2002; Jaffe and Hall, 2005). Whether the RhoAcypin pathway affects the actin cytoskeleton or specifically affects the microtubule cytoskeleton remains to be seen.

Does cypin protein exist in a complex with RhoA? So far, $>30$ effector proteins for Rho GTPases have been identified by affinity chromatography and the yeast two-hybrid screening system. These proteins interact specifically with the GTPbound form of the GTPase (Bishop and Hall, 2000). Using coimmunoprecipitation and affinity chromatography assays, we have been unable to detect cypin protein in a complex with either GDP- or GTP-bound RhoA (data not shown). Thus, protein-protein interactions between cypin and RhoA do not account for the effects of RhoA on cypin protein levels.

The most striking finding of our study is that activation of RhoA decreases cypin protein expression via a translationdependent mechanism (Fig. 7 $A, B$ ). In contrast, inhibition of RhoA does not affect cypin protein expression (Fig. 6). These data indicate that, when RhoA is in the resting or inactive state, it has no effect on arbor dendritic complexity and cypin protein levels; however, the increase in dendrite number by a dominant-negative RhoA mutant does occur through a cypindependent pathway (Fig. 5). In our model, GDP binding to RhoA does not allow RhoA to decrease cypin protein levels (Fig. 8). During activation of RhoA, GTP binds and RhoA regulates cypin protein levels via a translation-dependent mechanism (Figs. 7, 8). Thus, extracellular signals that regulate dendritic arborization can activate RhoA to inhibit dendrite branching by decreasing cypin protein expression.

There are many reports that neuronal activity is important for dendritic growth (Engert and Bonhoeffer, 1999; MaleticSavatic et al., 1999; Van Aelst and Cline, 2004). Specifically, NMDA receptor activation increases $\mathrm{Cdc} 42$ and Rac activity and decreases Rho activity required for the promotion of dendritic growth of Xenopus optic tectal neurons (Li et al., 2000, 2002). Furthermore, NMDA receptor activity is required for neurotrophin-promoted growth of cortical neuron dendrites (McAllister et al., 1995, 1997). We reported previously that treatment with $\mathrm{KCl}$ increases cypin protein expression in hippocampal neurons (Akum et al., 2004).
A
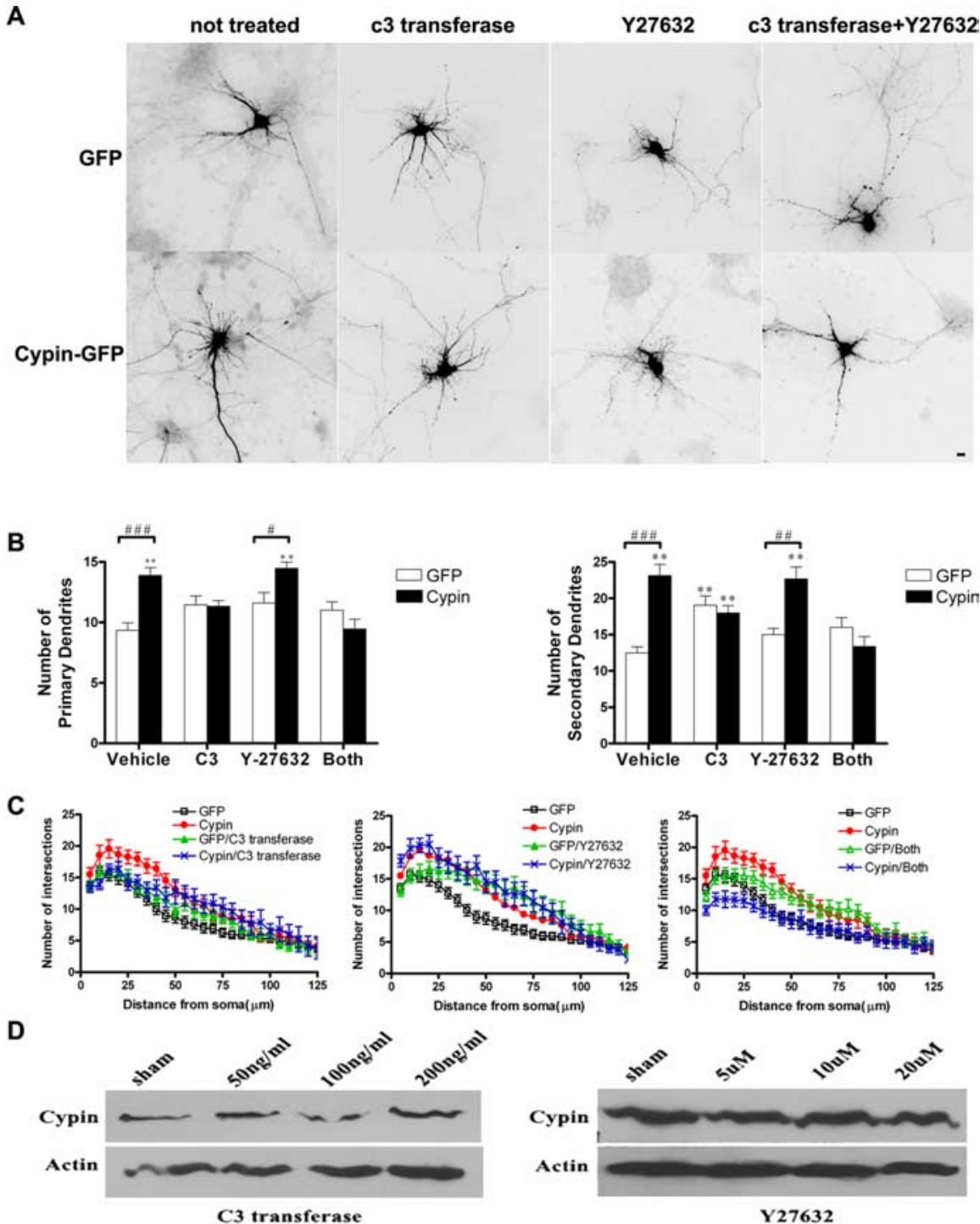

E
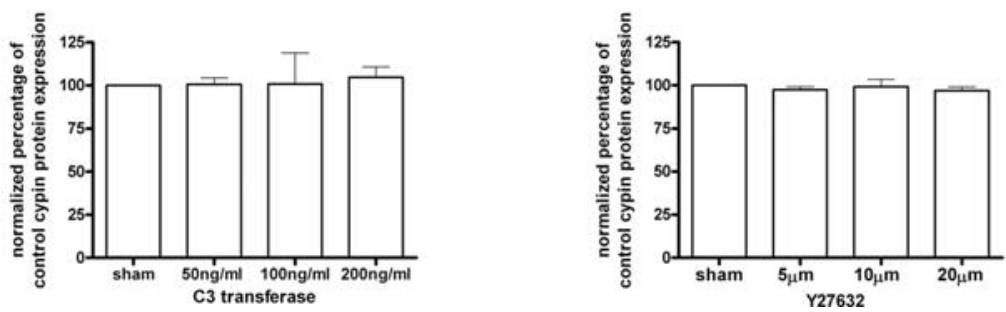

Figure 6. Treatment of neurons with $\mathrm{C} 3$ transferase, but not $\mathrm{Y} 27632$, blocks cypin-promoted increases in dendrite number without affecting cypin protein expression. Hippocampal neurons were transfected with constructs encoding GFP or cypin-GFP at DIV 10, treated with the appropriate drug at DIV 11 for $24 \mathrm{~h}$, and processed for immunostaining at DIV 12. A, GFP images of representative neurons. Scale bar, $10 \mu \mathrm{m} . \boldsymbol{B}$, Average number of primary and secondary dendrites in transfected neurons. $n$ values are as follows: in GFP group: none, $n=35 ; C 3, n=22 ; Y 27632, n=20 ;$ both, $n=18$; and in cypin group: none, $n=24 ; C 3, n=$ $15 ; Y 27632, n=15 ;$ both, $n=13$. ${ }^{\#} p<0.05,{ }^{\#} p<0.01$, \#\#\# $p 0.001$ by ANOVA followed by Bonferroni's multiple comparisons test. ${ }^{* *} p<0.01$ by ANOVA followed by Dunnett's multiple comparisons test compared with GFP. C, Left, Sholl analysis of hippocampal neurons. Overexpression of cypin significantly increases number of intersections in the range $20-55 \mu \mathrm{m}$ compared with expression of GFP (red circles; $p<0.01$ at $30-45 \mu \mathrm{m}, p<0.05$ at other points). Treatment with 33 transferase does not change dendritic arbor complexity, even combined with expression of cypin (blue $\mathrm{x}$ ). Middle, Neurons overexpressing cypin and treated with $\mathrm{Y} 27632$ (blue $\mathrm{x}$ ) show a significant increase in number of intersections at $5-75 \mu \mathrm{m}$ from the soma (except at $10 \mu \mathrm{m} ; p<0.05$ at $15,25-35,60$, and $75 \mu \mathrm{m}, p<0.01$ at other points). Neurons treated with Y27632 (green triangles) alone show increased number of intersections at $35-75 \mu \mathrm{m}$ from the soma ( $p<0.05$ at 35 and $75 \mu \mathrm{m}, p<0.01$ at other points). $p$ values were determined by Kruskal-Wallis test followed by Dunn's multiple comparisons test compared with GFP. Right, Neurons overexpressing cypin (blue $\mathrm{x}$ ) or GFP (green triangles) and treated with both inhibitors show no significant change in number of intersections from that of control (GFP). D, Hippocampal neurons were treated with C3 transferase or Y27632 at indicated concentrations at DIV 11 for $24 \mathrm{~h}$. Representative Western blot of three individual experiments is shown. $E$, Quantitative analysis shows that treatments do not change is cypin protein expression ( $p>0.05$ by ANOVA). Error bars indicate SEM. 
A

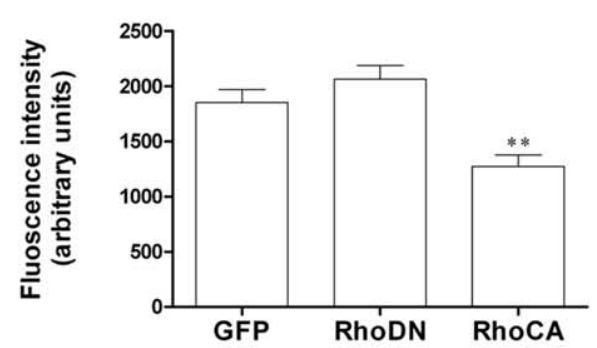

B
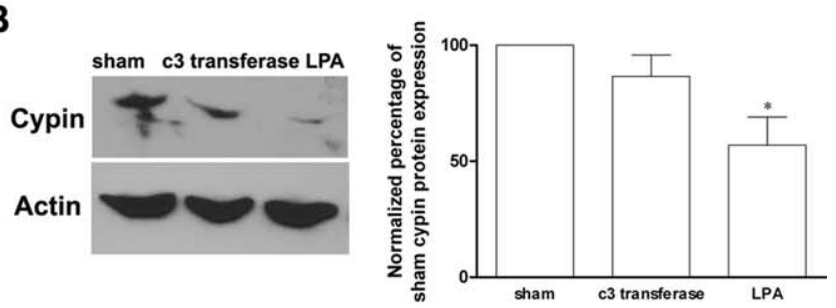

C

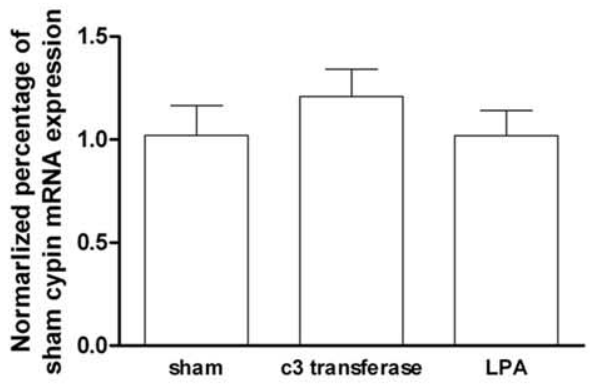

D

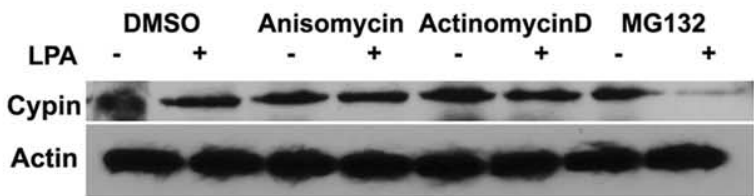

E

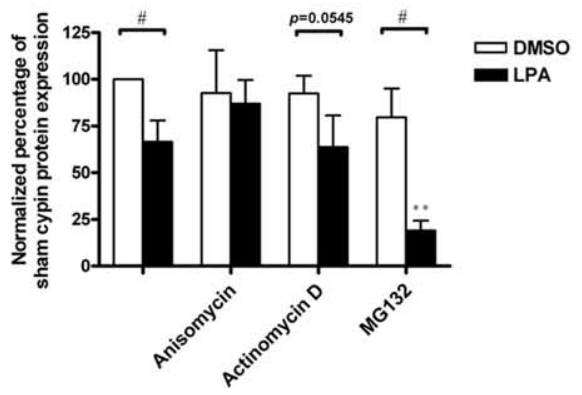

Figure 7. Activation of RhoA decreases cypin protein expression via a translation-dependent mechanism. $\boldsymbol{A}$, Cultured hippocampal neurons were transfected with cDNAs encoding RhoCA or RhoDN at DIV 10. Neurons were fixed and immunostained at DIV 12, and cypin levels were quantitated using ImagePro software. $n$ values are as follows: GFP, $n=30$; RhoCA, $n=36$; RhoDN, $n=35$. ${ }^{* *} p<0.01$ by ANOVA followed by Dunnett's multiple comparison test compared with GFP. B, Hippocampal neurons were treated with $100 \mathrm{ng} / \mathrm{ml} \mathrm{C3}$ transferase at DIV 11 for $24 \mathrm{~h}$ or $1 \mu \mathrm{g} / \mathrm{ml}$ LPA at DIV 12 for $15 \mathrm{~min}$. Quantitation of Western blots shows that there is significant change in cypin protein expression with LPA but not C 3 transferase treatment $(n=$ 3). ${ }^{*} p<0.05$ by ANOVA followed by Dunnett's multiple comparison test compared with GFP.C, Semiquantitative real-time reverse transcription-PCR shows there is no significant change in cypin mRNA levels with these treatments. D, At DIV 12, hippocampal neurons were preincubated with $40 \mu \mathrm{m}$ anisomycin, $5 \mu \mathrm{m}$ actinomycin D, or $50 \mu \mathrm{m}$ MG132 for $30 \mathrm{~min}$. The neurons were then treated with LPA at $1 \mu \mathrm{g} / \mathrm{ml}$ for $15 \mathrm{~min}$. A representative blot is shown from four experiments. $\boldsymbol{E}$, Quantitation of Western blots shows that anisomycin, but not actinomycin D or MG132, blocks the LPA-mediated decrease in cypin protein expression. ${ }^{* *} p<0.01$ by ANOVA followed by Dunnett's multiple comparison test compared with DMSO; ${ }^{*} p<0.05$ by paired $t$ test $(n=4)$. Error bars indicate SEM.

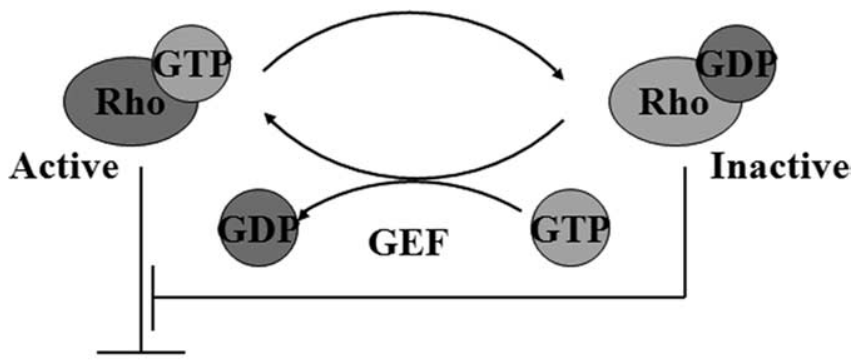

Translation of cypin
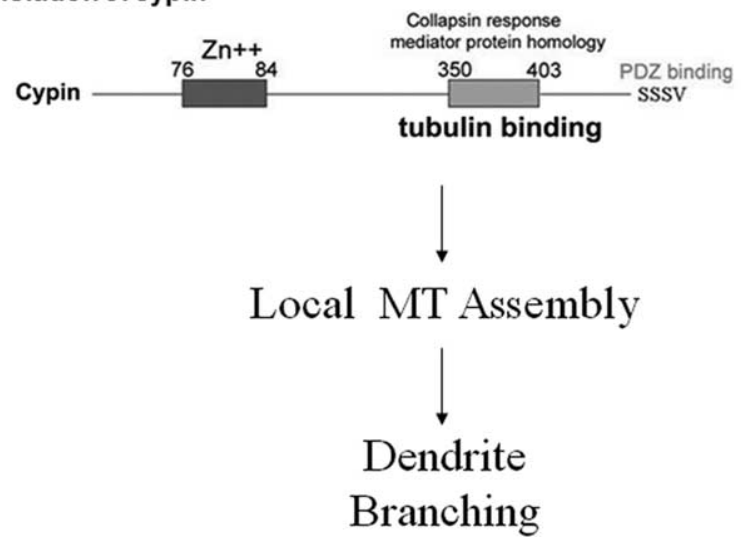

Figure 8. Model for RhoA-regulated dendrite branching. Activation of RhoA decreases cypin protein expression and in turn inhibits local microtubule (MT) assembly and decreases dendrite branching. Inhibition of RhoA will allow cypin protein levels to remain at a constitutive state and disallow the inhibitory effect of activated RhoA on cypin translation and dendrite branching.

Together, our data and reports in the literature suggest that a common pathway including neuronal activity, RhoA, and cypin exists to regulate dendritic arborization.

What is special about cypin? Why does general stabilization of microtubules with agents such as taxol not increase dendrite branching? Our recent reports suggest that the promotion of local assembly of microtubules rather than global assembly is key. We recently showed that overexpression of PSD-95, a PDZ (PSD95/Discs large/zona occludens-1)-containing postsynaptic scaffolding protein that binds to cypin, decreases higher-order dendrite number, and stops cypin-mediated dendritic branching (Charych et al., 2006). Furthermore, the binding of cypin to PSD-95 is essential for stabilizing cypin-promoted increases in dendrite number, suggesting that PSD-95 may localize cypin to sites for dendritic branching (Charych et al., 2006). Cypin would then remove PSD-95, and hence the block on dendrite branching, and promote local microtubule assembly. Similarly, snapin, another cypin binding partner, competes with tubulin heterodimers to bind to cypin and slows cypin-mediated microtubule assembly (Chen et al., 2005). Overexpression of snapin decreases primary dendrite number but not secondary dendrite number (Chen et al., 2005). Snapin protein levels are higher in the cell body than in the dendrites of developing hippocampal neurons, and cypin is expressed throughout the cell body and processes (Firestein et al., 1999; Akum et al., 2004; Chen et al., 2005). This differential expression of snapin causes changes only in primary and not secondary dendrite number (Chen et al., 2005), again suggesting that the promotion of microtubule assembly locally regulates dendrite branching. Most studies have focused on axonal branching and growth cone guidance and that microtubule transport or changes in microtubule stability underlie these processes (Baas et al., 2005; Kalil and Dent, 2005). Given 
that cypin promotes microtubule assembly and is downstream of RhoA, we believe that we have identified a novel pathway by which RhoA acts to regulate dendrite number by altering the microtubule cytoskeleton.

\section{References}

Ahnert-Hilger G, Holtje M, Grosse G, Pickert G, Mucke C, NixdorfBergweiler B, Boquet P, Hofmann F, Just I (2004) Differential effects of Rho GTPases on axonal and dendritic development in hippocampal neurones. J Neurochem 90:9-18.

Akum BF, Chen M, Gunderson SI, Riefler GM, Scerri-Hansen MM, Firestein BL (2004) Cypin regulates dendrite patterning in hippocampal neurons by promoting microtubule assembly. Nat Neurosci 7:145-152.

Arimura N, Kaibuchi K (2005) Key regulators in neuronal polarity. Neuron 48:881-884.

Baas PW, Karabay A, Qiang L (2005) Microtubules cut and run. Trends Cell Biol 15:518-524.

Bishop AL, Hall A (2000) Rho GTPases and their effector proteins. Biochem J 348:241-255.

Charest PG, Firtel RA (2007) Big roles for small GTPases in the control of directed cell movement. Biochem J 401:377-390.

Charych EI, Akum BF, Goldberg JS, Jornsten RJ, Rongo C, Zheng JQ, Firestein BL (2006) Activity-independent regulation of dendrite patterning by postsynaptic density protein PSD-95. J Neurosci 26:10164-10176.

Chen M, Lucas KG, Akum BF, Balasingam G, Stawicki TM, Provost JM, Riefler GM, Jornsten RJ, Firestein BL (2005) A novel role for snapin in dendrite patterning: interaction with cypin. Mol Biol Cell 16:5103-5114.

Cui R, Tieu B, Recinos A, Tilton RG, Brasier AR (2006) RhoA mediates angiotensin II-induced phospho-Ser536 nuclear factor kappaB/RelA subunit exchange on the interleukin-6 promoter in VSMCs. Circ Res 99:723-730

Dent EW, Barnes AM, Tang F, Kalil K (2004) Netrin-1 and semaphorin 3A promote or inhibit cortical axon branching, respectively, by reorganization of the cytoskeleton. J Neurosci 24:3002-3012.

Engert F, Bonhoeffer T (1999) Dendritic spine changes associated with hippocampal long-term synaptic plasticity. Nature 399:66-70.

Etienne-Manneville S, Hall A (2002) Rho GTPases in cell biology. Nature 420:629-635.

Firestein BL, Brenman JE, Aoki C, Sanchez-Perez AM, El-Husseini AE, Bredt DS (1999) Cypin: a cytosolic regulator of PSD-95 postsynaptic targeting. Neuron 24:659-672.

Fukata Y, Itoh TJ, Kimura T, Menager C, Nishimura T, Shiromizu T, Watanabe $\mathrm{H}$, Inagaki $\mathrm{N}$, Iwamatsu $\mathrm{A}$, Hotani $\mathrm{H}$, Kaibuchi $\mathrm{K}$ (2002) CRMP-2 binds to tubulin heterodimers to promote microtubule assembly. Nat Cell Biol 4:583-591.

Gebbink MF, Kranenburg O, Poland M, van Horck FP, Houssa B, Moolenaar WH (1997) Identification of a novel, putative Rho-specific GDP/GTP exchange factor and a RhoA-binding protein: control of neuronal morphology. J Cell Biol 137:1603-1613.

Govek EE, Newey SE, Van Aelst L (2005) The role of the Rho GTPases in neuronal development. Genes Dev 19:1-49.

Hall A (1998) Rho GTPases and the actin cytoskeleton. Science 279:509-514.

Hoogenraad CC, Milstein AD, Ethell IM, Henkemeyer M, Sheng M (2005) GRIP1 controls dendrite morphogenesis by regulating EphB receptor trafficking. Nat Neurosci 8:906-915.

Ishizaki T, Morishima Y, Okamoto M, Furuyashiki T, Kato T, Narumiya S (2001) Coordination of microtubules and the actin cytoskeleton by the Rho effector mDial. Nat Cell Biol 3:8-14

Jaffe AB, Hall A (2005) Rho GTPases: biochemistry and biology. Annu Rev Cell Dev Biol 21:247-269.

Jaworski J, Spangler S, Seeburg DP, Hoogenraad CC, Sheng M (2005) Control of dendritic arborization by the phosphoinositide-3'-kinase-Aktmammalian target of rapamycin pathway. J Neurosci 25:11300-11312.

Kalil K, Dent EW (2005) Touch and go: guidance cues signal to the growth cone cytoskeleton. Curr Opin Neurobiol 15:521-526.

Larsson E, Zhou X, Akyurek LM (2007) RhoA-dependent vascular smooth muscle cell-specific transcription: adding diaphanous formins to the puzzle. Arterioscler Thromb Vasc Biol 27:448-449.

Lee T, Winter C, Marticke SS, Lee A, Luo L (2000) Essential roles of Dro- sophila RhoA in the regulation of neuroblast proliferation and dendritic but not axonal morphogenesis. Neuron 25:307-316.

Li Z, Van Aelst L, Cline HT (2000) Rho GTPases regulate distinct aspects of dendritic arbor growth in Xenopus central neurons in vivo. Nat Neurosci 3:217-225.

Li Z, Aizenman CD, Cline HT (2002) Regulation of rho GTPases by crosstalk and neuronal activity in vivo. Neuron 33:741-750.

Livak KJ, Schmittgen TD (2001) Analysis of relative gene expression data using real-time quantitative PCR and the 2(-Delta Delta C (T)) method. Methods 25:402-408.

Luo L (2002) Actin cytoskeleton regulation in neuronal morphogenesis and structural plasticity. Annu Rev Cell Dev Biol 18:601-635.

Maekawa M, Ishizaki T, Boku S, Watanabe N, Fujita A, Iwamatsu A, Obinata T, Ohashi K, Mizuno K, Narumiya S (1999) Signaling from Rho to the actin cytoskeleton through protein kinases ROCK and LIM-kinase. Science 285:895-898.

Maletic-Savatic M, Malinow R, Svoboda K (1999) Rapid dendritic morphogenesis in CA1 hippocampal dendrites induced by synaptic activity. Science 283:1923-1927.

McAllister AK, Lo DC, Katz LC (1995) Neurotrophins regulate dendritic growth in developing visual cortex. Neuron 15:791-803.

McAllister AK, Katz LC, Lo DC (1997) Opposing roles for endogenous BDNF and NT-3 in regulating cortical dendritic growth. Neuron 18:767-778.

Nakayama AY, Harms MB, Luo L (2000) Small GTPases Rac and Rho in the maintenance of dendritic spines and branches in hippocampal pyramidal neurons. J Neurosci 20:5329-5338.

Newey SE, Velamoor V, Govek EE, Van Aelst L (2005) Rho GTPases, dendritic structure, and mental retardation. J Neurobiol 64:58-74.

Nguyen Ba-Charvet KT, Brose K, Ma L, Wang KH, Marillat V, Sotelo C, Tessier-Lavigne M, Chedotal A (2001) Diversity and specificity of actions of Slit2 proteolytic fragments in axon guidance. J Neurosci 21:4281-4289.

Palazzo AF, Cook TA, Alberts AS, Gundersen GG (2001) mDia mediates Rho-regulated formation and orientation of stable microtubules. Nat Cell Biol 3:723-729.

Pilpel Y, Segal M (2004) Activation of PKC induces rapid morphological plasticity in dendrites of hippocampal neurons via Rac and Rhodependent mechanisms. Eur J Neurosci 19:3151-3164.

Redmond L, Ghosh A (2001) The role of Notch and Rho GTPase signaling in the control of dendritic development. Curr Opin Neurobiol 11:111-117.

Ren XD, Kiosses WB, Schwartz MA (1999) Regulation of the small GTPbinding protein Rho by cell adhesion and the cytoskeleton. EMBO J 18:578-585.

Riento K, Ridley AJ (2003) Rocks: multifunctional kinases in cell behaviour. Nat Rev Mol Cell Biol 4:446-456.

Ruchhoeft ML, Ohnuma S, McNeill L, Holt CE, Harris WA (1999) The neuronal architecture of Xenopus retinal ganglion cells is sculpted by rhofamily GTPases in vivo. J Neurosci 19:8454-8463.

Sin WC, Haas K, Ruthazer ES, Cline HT (2002) Dendrite growth increased by visual activity requires NMDA receptor and Rho GTPases. Nature 419:475-480.

Threadgill R, Bobb K, Ghosh A (1997) Regulation of dendritic growth and remodeling by Rho, Rac, and Cdc42. Neuron 19:625-634.

Van Aelst L, Cline HT (2004) Rho GTPases and activity-dependent dendrite development. Curr Opin Neurobiol 14:297-304.

Wadsworth WG (2002) Moving around in a worm: netrin UNC-6 and circumferential axon guidance in C. elegans. Trends Neurosci 25:423-429.

Watanabe T, Noritake J, Kaibuchi K (2005) Regulation of microtubules in cell migration. Trends Cell Biol 15:76-83.

Wong WT, Faulkner-Jones BE, Sanes JR, Wong RO (2000) Rapid dendritic remodeling in the developing retina: dependence on neurotransmission and reciprocal regulation by Rac and Rho. J Neurosci 20:5024-5036.

Yu X, Malenka RC (2003) Beta-catenin is critical for dendritic morphogenesis. Nat Neurosci 6:1169-1177.

Yuan G, Bin JC, McKay DJ, Snyder FF (1999) Cloning and characterization of human guanine deaminase. Purification and partial amino acid sequence of the mouse protein. J Biol Chem 274:8175-8180.

Zheng Y (2004) G protein control of microtubule assembly. Annu Rev Cell Dev Biol 20:867-894. 